\title{
Anomalous tracer diffusion in hard-sphere suspensions
}

\author{
Stephen S. L. Peppin
}

\begin{abstract}
Coupled equations describing diffusion and cross-diffusion of tracer particles in hard-sphere suspensions are derived and solved numerically. In concentrated systems with strong excluded volume and viscous interactions the tracer motion is subdiffusive. Cross diffusion generates transient perturbations to the host-particle matrix, which affect the motion of the tracer particles leading to nonlinear mean squared displacements. Above a critical host-matrix concentration the tracers experience clustering and uphill diffusion, moving in opposition to their own concentration gradient. A linear stability analysis indicates that cross diffusion can lead to unstable concentration fluctuations in the suspension. The instability is a potential mechanism for the appearance of dynamic and structural heterogeneity in suspensions near the glass transition.
\end{abstract}

\section{Introduction}

In complex fluids and colloidal suspensions the diffusion of tracer particles often cannot be described by classical theories of Brownian motion $[1,2]$. In concentrated suspensions the mean squared displacement of the tracer particles is a nonlinear function of time, and the probability distribution function is non-Gaussian, owing to hydrodynamic and spatial heterogeneity in the host matrix [1,3-13]. In order to explore such effects within the generic context of hard-sphere suspensions, experimental methods such as dynamic light scattering [3] and particle tracking [6] have been used extensively over the past several decades, as well as molecular dynamics simulations [13] and statistical mechanical models $[1,2]$. At high concentrations near the glass transition long-range viscous interactions between the particles make accurate simulations and experiments challenging, and there is uncertainty about the origin of the spatial and temporal heterogeneity within the host matrix $[6,12-16]$.

In this work a continuum model of anomalous diffusion in hard-sphere suspensions is developed, in which the differential equations governing diffusion and cross-diffusion of tracer particles in concentrated suspensions are solved numerically. The model simulates the development of spatiotemporal heterogeneity within the host matrix caused by the initial tracer distribution, and the effect of this on the motion of the tracer particles. This yields a macroscopic view of the coupling effects between the host matrix and tracer particles, and the onset of anomalous tracer diffusion.

In Section 2 the flux equations describing diffusion and cross-diffusion in suspensions are briefly reviewed, and in Section 3 the governing conservation equations are described and put in dimensionless form. The equations are solved in Section 4 for the case of a Gaussian tracer pulse placed within an initially uniform host matrix, as depicted in figure 1. The macroscopic shape and time behaviour of the perturbation to the host matrix caused by the tracer particles is simulated, as well as the feedback effect on the tracer motion. Section 4.2 contains a discussion of the results and their relation to light scattering and particle tracking experiments. Finally, in Section 5 a linear stability analysis of the governing equations is undertaken, showing that near the glass transition an initially uniform suspension is marginally stable to the growth of normal mode concentration fluctuations.

For God so loved the world, that He gave His only begotten Son, that whosoever believeth in Him should not perish, but have everlasting life. For God sent not His Son into the world to condemn the world; but that the world through Him might be saved. John 3:16-17 


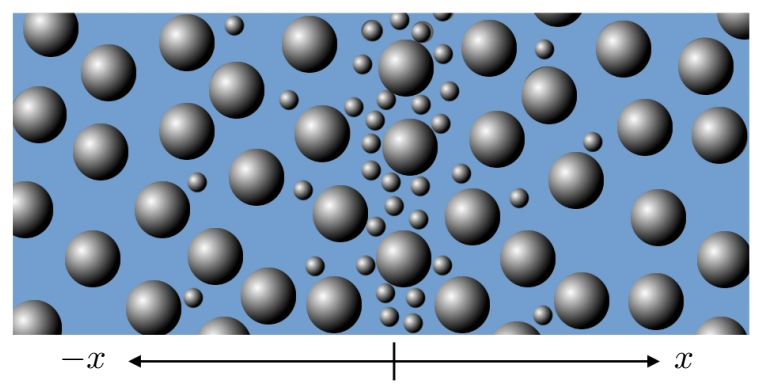

Figure 1: Schematic of a Gaussian pulse of hard-sphere tracer particles centred at $x=0$ within a uniform suspension of larger host particles.

\section{Diffusion and cross-diffusion in concentrated suspensions}

The system to be studied is illustrated in figure 1, in which a uniform suspension of spherical host particles of radius $R_{1}$ contains a Gaussian pulse of smaller tracer particles of radius $R_{2}$ within the pore space. The flux equations describing diffusion and cross-diffusion of the host and tracer particles in the one-dimensional system in figure 1 can be written as

$$
\begin{aligned}
& J_{1}=-D_{11} \frac{\partial n_{1}}{\partial x}-D_{12} \frac{\partial n_{2}}{\partial x}, \\
& J_{2}=-D_{21} \frac{\partial n_{1}}{\partial x}-D_{22} \frac{\partial n_{2}}{\partial x},
\end{aligned}
$$

where $n_{1}$ and $n_{2}$ are the concentrations of the host and tracer particles (number of particles per unit volume of mixture) [17]. In quiescent systems with zero volume velocity the host particle flux is $J_{1}=n_{1} u_{1}$, where $u_{1}$ is the average host particle velocity, and $J_{2}=n_{2} u_{2}$ is the flux of tracer particles. In equations (1) $D_{11}$ and $D_{22}$ are the Fickian diffusion coefficients of the host and tracer particles, respectively, while $D_{12}$ and $D_{21}$ are cross-diffusion coefficients. Phenomenological expressions for the diffusion and cross-diffusion coefficients as functions of concentration have been obtained previously [18], and are briefly summarized below.

\subsection{Diffusion coefficients}

In the dilute tracer limit $n_{2} \rightarrow 0$, the diffusion and cross-diffusion coefficients in concentrated hard-sphere suspensions can be written in the approximate form

$$
\begin{gathered}
D_{11}=D_{1}^{0} K \hat{\Pi}_{1}, \quad D_{12}=D_{1}^{0} K \sigma / \alpha, \\
D_{21}=D_{2}^{0}\left(\hat{\ell} K \hat{\Pi}_{1}-\gamma \tau\right), \quad D_{22}=D_{2}^{0} \tau,
\end{gathered}
$$

where $D_{i}^{0}=k_{B} T /\left(6 \pi R_{i} \eta_{0}\right)$ is the Stokes-Einstein diffusivity of particle $i, k_{B}$ is Boltzmann's constant, $T$ is temperature, and $\eta_{0}$ is the viscosity of the suspending fluid [18]. In $(2 a) K$ is the dimensionless permeability (viscous mobility) of the host-particle matrix and $\hat{\Pi}_{1}=\Pi_{1} /\left(k_{B} T\right)=$ $\mathrm{d}\left(\phi_{1} Z\right) / \mathrm{d} \phi_{1}$, where $\Pi_{1}=\left(\partial \Pi / \partial n_{1}\right)_{T}$ is the derivative of the host-matrix osmotic pressure $\Pi$ with respect to concentration; $\phi_{1}=n_{1} v_{1}$ is the host-particle volume fraction, $v_{1}=\frac{4}{3} \pi R_{1}^{3}$ is the volume of a host particle and $Z\left(\phi_{1}\right)$ is the host-matrix compressiblity factor. In $(2 b) \sigma$ is the reflection coefficient of the host matrix and $\alpha$ is the equilibrium partition coefficient of the tracer particles between the pore space and the bulk fluid. In $(2 c) \gamma$ is a preferential interaction coefficient accounting for excluded volume effects and $\hat{\ell}=\phi_{2} \ell /\left(\lambda^{2} \phi_{1}\right)$, where $\phi_{2}=n_{2} v_{2}$ is the tracer-particle volume fraction, $\lambda=R_{2} / R_{1}$ is the ratio of particle sizes and $\ell=(\alpha+\sigma-1) / \alpha$ is a viscous cross-diffusion factor between the tracer and host particles. Finally, in $(2 d) \tau$ is the diffusive tortuosity factor accounting for viscous hindrance to tracer diffusion within the pore space. 
For spherical particles approximate expressions for the friction and compressibility factors of the host-particle matrix are

$$
K=\left(1-\phi_{1}\right)^{6.55} \quad \text { and } \quad Z=\frac{1+a \phi_{1}}{1-\phi_{1} / \phi_{p}}
$$

where $a=4-1 / \phi_{p}$ and $\phi_{p}=0.64$ is the volume fraction at random close packing. More accurate expressions for $K$ and $Z$ are available [18]; however, equations (3) have a relatively simple form similar to that used recently by Worster et al. [19] and will suffice for qualitative purposes. In the dilute limit $\phi_{1} \ll 1$ equations (3) reduce to the exact results $K=1-6.55 \phi_{1}$ and $Z=1+4 \phi_{1}[20,21]$. Given $Z, \hat{\Pi}_{1}$ can be obtained as

$$
\hat{\Pi}_{1}=\frac{\mathrm{d}\left(\phi_{1} Z\right)}{\mathrm{d} \phi_{1}}=\frac{1+2 a \phi_{1}-a \phi_{1}^{2} / \phi_{p}}{\left(1-\phi_{1} / \phi_{p}\right)^{2}} .
$$

Approximate expressions for the partition coefficient $\alpha$ and preferential interaction coefficient $\gamma$ in hard-sphere suspensions are

$$
\alpha=\mathrm{e}^{-b_{12} \phi_{1}} \quad \text { and } \quad \gamma=-n_{2} b_{12} v_{1}=-\phi_{2} b_{12} / \lambda^{3},
$$

where $\lambda=R_{2} / R_{1}$ is the size ratio of the tracer and host particles, $b_{12}=(1+\lambda)^{3}$ is a thermodynamic coupling coefficient, $\phi_{2}=n_{2} v_{2}$ is the tracer volume fraction and $v_{2}=\frac{4}{3} \pi R_{2}^{3}$ is the tracer particle volume. In the limit $\phi_{1} \rightarrow 0$ and $\lambda \rightarrow 0,(5 a)$ shows that the partition coefficient is equal to the void fraction, $\alpha=1-\phi_{1}$. A more general expression for $\alpha$ is obtained in the Appendix, where it is shown that equation $(5 a)$ gives qualitatively correct results for all $\phi_{1}$ when $\lambda \lesssim 1$.

Power law constitutive equations for the diffusive tortuosity factor $\tau$ and reflection coefficient $\sigma$ in hard sphere suspensions are

$$
\tau=\left(1-\lambda_{d}\right)^{a_{\tau}} \quad \text { and } \quad \sigma=1-\left(1-\lambda_{d}\right)^{a_{\sigma}},
$$

where

$$
\lambda_{d}=\frac{3 \lambda \phi_{1}}{c_{d}\left(1-\phi_{1}\right)} \quad \text { and } \quad c_{d}=3 \frac{\phi_{g}}{\left(1-\phi_{g}\right)} \frac{\left(1+\lambda^{2}\right)}{(1+\lambda)} .
$$

Here $\lambda_{d}$ is the ratio of the tracer size $R_{2}$ to the locally averaged pore size within the suspension, $R_{d}=c_{d} R_{p}$, where $R_{p}=3 \phi_{1} /\left[R_{1}\left(1-\phi_{1}\right)\right]$ is the physical pore size [22]. The quantity $c_{d}$ is a scaling factor accounting for fluctuations in the pore size and $\phi_{g}=0.58$ is the volume fraction at the hard-sphere glass transition. The scaling factor ensures that $\tau \rightarrow \eta_{0} / \eta$ in the large tracer limit $R_{2} \rightarrow \infty$, where $\eta\left(\phi_{1}\right)$ is the dynamic viscosity of the host suspension. When $\lambda_{d} \geq 1$ the tracer particles are trapped in the pores and $\tau=0$ while $\sigma=1$. The exponents $a_{\tau}$ and $a_{\sigma}$ in (6) are

$$
a_{\tau}=\frac{\tau_{1} c_{d}}{3 \lambda} \quad \text { and } \quad a_{\sigma}=\frac{\sigma_{1} c_{d}}{3 \lambda}
$$

where $\tau_{1}=2.5 \lambda /(.22+\lambda)$ and $\sigma_{1}=b_{12}-\left(1+3 \lambda+\lambda^{2}\right)+\lambda^{2} /\left(1+\lambda^{3}\right)$. Finally, given $\alpha$ and $\sigma$, the cross-diffusion factor can be obtained as

$$
\ell=\frac{(\sigma+\alpha-1)}{\alpha} \quad \text { and } \quad \hat{\ell}=\frac{\phi_{2} \ell}{\lambda^{2} \phi_{1}}
$$

With equations (3)-(9), the diffusion coefficients in (2) can be determined as functions of the volume fractions $\phi_{1}$ and $\phi_{2}$ and the particle size ratio $\lambda$.

\section{Governing equations}

In figure 1 a Gaussian tracer pulse of width $d$ and concentration $n_{2}=n_{2}^{0} \mathrm{e}^{-x^{2} / 2 d^{2}}$ is placed at $t=0$ within a uniform matrix of the host particles at initial concentration $n_{1}^{0}$. The flux equations (1) can be combined with conservation of particle number,

$$
\frac{\partial n_{i}}{\partial t}+\frac{\partial n_{i} u_{i}}{\partial x}=0 \quad(i=1,2)
$$


to yield two coupled diffusion equations of the form

$$
\begin{aligned}
& \frac{\partial n_{1}}{\partial t}=\frac{\partial}{\partial x}\left(D_{11} \frac{\partial n_{1}}{\partial x}+D_{12} \frac{\partial n_{2}}{\partial x}\right), \\
& \frac{\partial n_{2}}{\partial t}=\frac{\partial}{\partial x}\left(D_{21} \frac{\partial n_{1}}{\partial x}+D_{22} \frac{\partial n_{2}}{\partial x}\right) .
\end{aligned}
$$

Upon replacing the number densities with volume fractions using the relation $\phi_{i}=n_{i} v_{i}$, and introducing the dimensionless length and time scales, $\hat{x}=x / d$ and $\hat{t}=t D_{2}^{0} / d^{2}$, equations (11) can be written in dimensionless form as

$$
\begin{gathered}
\frac{\partial \phi_{1}}{\partial \hat{t}}=\lambda \frac{\partial}{\partial \hat{x}}\left(\hat{D}_{11} \frac{\partial \phi_{1}}{\partial \hat{x}}+\lambda^{-3} \hat{D}_{12} \frac{\partial \phi_{2}}{\partial \hat{x}}\right), \\
\frac{\partial \phi_{2}}{\partial \hat{t}}=\frac{\partial}{\partial \hat{x}}\left(\lambda^{3} \hat{D}_{21} \frac{\partial \phi_{1}}{\partial \hat{x}}+\hat{D}_{22} \frac{\partial \phi_{2}}{\partial \hat{x}}\right),
\end{gathered}
$$

where $\hat{D}_{i j}=D_{i j} / D_{i}^{0}$ and $\lambda=R_{2} / R_{1}=D_{1}^{0} / D_{2}^{0}$.

\subsection{Initial and boundary conditions}

At $\hat{t}=0$ the host suspension is of uniform volume fraction $\phi_{1}^{0}$ giving the initial conditions

$$
\phi_{1}=\phi_{1}^{0} \quad \text { and } \quad \phi_{2}=\phi_{2}^{0} \mathrm{e}^{-\hat{x}^{2} / 2} \quad(-\infty<\hat{x}<\infty) .
$$

The boundary conditions are

$$
\phi_{1} \rightarrow \phi_{1}^{0} \quad \text { and } \quad \phi_{2} \rightarrow 0 \quad(\hat{x} \rightarrow \pm \infty) .
$$

Equations (12)-(14) depend on three dimensionless parameters - the initial volume fractions $\phi_{1}^{0}$, $\phi_{2}^{0}$, and the particle size ratio $\lambda$.

\section{Results}

Equations (12)-(14) were solved using Matlab with a method of lines routine developed by Scheisser and Griffiths [23] (the Matlab files are included as Supplemental Material). Figure 2 shows profiles of $\phi_{i}(\hat{x}, \hat{t})$ for the case $\lambda=0.75$ with initial volume fractions corresponding to a concentrated host matrix $\left(\phi_{1}^{0}=0.59\right)$ and dilute tracer distribution $\left(\phi_{2}^{0}=0.01\right)$. The solid curves in figure $2 a$ are the computed tracer volume fraction profiles at $\hat{t}=0.1$ (blue) and $\hat{t}=100$ (red), while the dashed curves are the Gaussian profiles obtained by setting the cross-diffusion terms to zero $\left(\hat{D}_{12}=\hat{D}_{21}=0\right)$. In the absence of cross-diffusion equation $(12 b)$ has the analytical Gaussian solution

$$
\phi_{2}(\hat{x}, \hat{t})=\frac{\phi_{2}^{0}}{\sqrt{1+2 \hat{D}_{22} t}} \exp \left(-\frac{\hat{x}^{2} / 2}{1+2 \hat{D}_{22} t}\right),
$$

which also gives the dashed curves in figure $2 a$. At $\hat{t}=0.1$ the solid and dashed curves overlap, while at $\hat{t}=100$ the tracer concentration profile departs from the Gaussian solution.

The physical origin of the non-Gaussian diffusion at $\hat{t}=100$ is illustrated in figure $2 b$, which shows the host-matrix volume fraction profiles computed from equation $(12 a)$ at $\hat{t}=0.1$ (blue), $\hat{t}=1$ (orange) and $\hat{t}=100$ (red). Collective motion of the host particles, induced by the initial tracer pulse through the cross-diffusion term in $(12 a)$, leads to a transient perturbation of the host-matrix volume fraction in the vicinity of $\hat{x}=0$. This $\phi_{1}(\hat{x}, \hat{t})$ profile in turn affects the tracer particle's motion via the cross-diffusion term in (12b). 

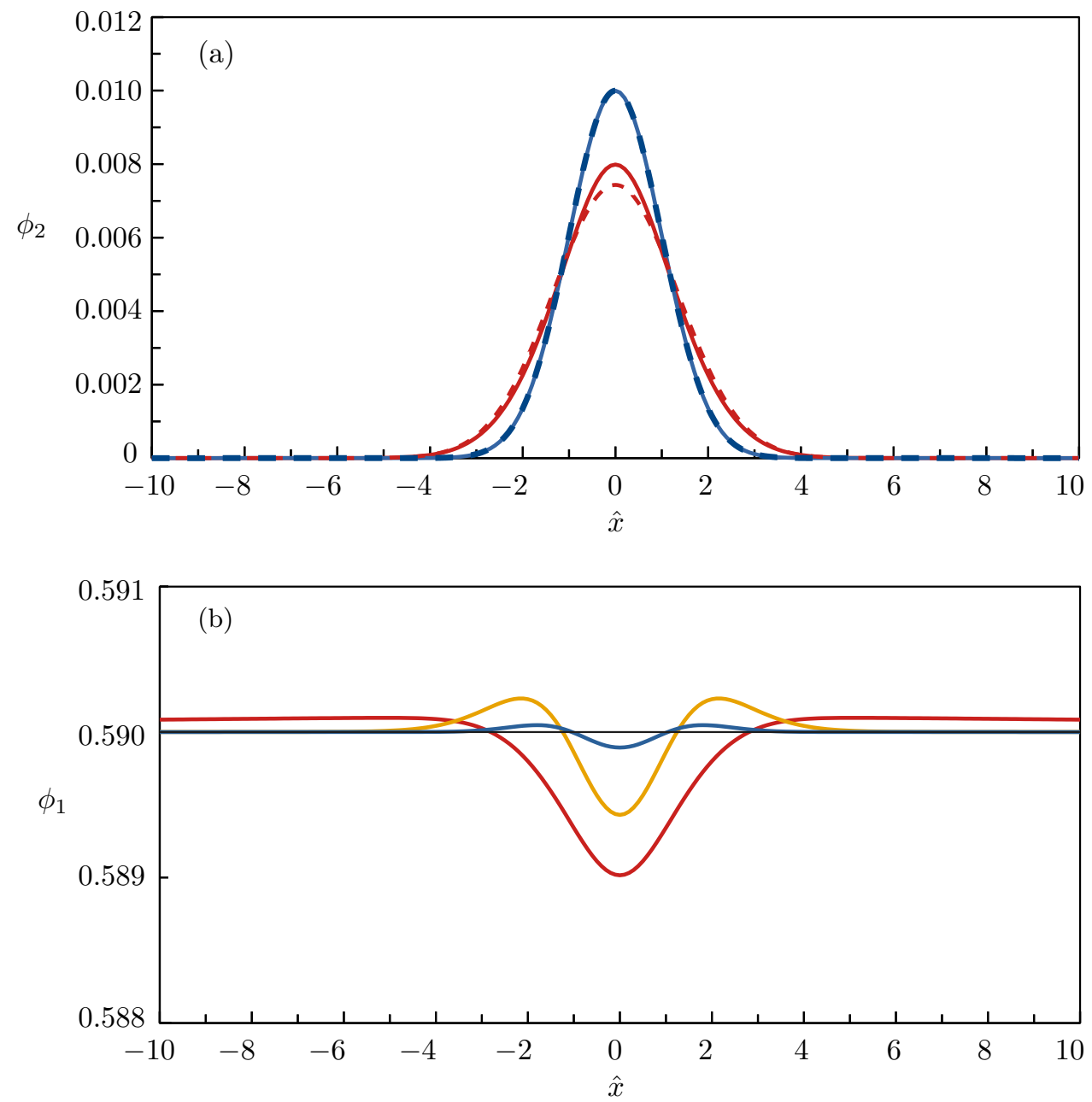

Figure 2: Plots of (a) tracer volume fraction $\phi_{2}(\hat{x})$ and (b) host matrix volume fraction $\phi_{1}(\hat{x})$ at times $\hat{t}=0.01$ (blue), $\hat{t}=0.1$ (orange) and $\hat{t}=100$ (red) for the case $\phi_{1}^{0}=0.59, \phi_{2}^{0}=0.01$ and $\lambda=0.75$. The dashed curves in (a) are the Gaussian $\phi_{2}$ profiles obtained in the absence of cross-diffusion.

\subsection{Tracer mean squared displacement}

Figure 3 shows the tracer mean squared displacement (MSD), calculated as

$$
\left\langle\hat{x}(\hat{t})^{2}\right\rangle=\int_{-L}^{L} \hat{x}^{2}[P(\hat{x}, \hat{t})-P(\hat{x}, 0)] \mathrm{d} \hat{x},
$$

where $2 L$ is the domain size and $P(\hat{x}, \hat{t})=\phi_{2}(\hat{x}, \hat{t}) /\left(\phi_{2}^{0} \sqrt{2 \pi}\right)$ is the tracer probability density function (the $\sqrt{2 \pi}$ factor ensures that $\int_{-\infty}^{\infty} P(\hat{x}, \hat{t}) \mathrm{d} \hat{x}=1$ ). The dashed lines show the linear MSD, $\left\langle\hat{x}^{2}\right\rangle=2 \hat{D}_{22} \hat{t}$, obtained in the absence of cross diffusion. At early times $\hat{t} \lesssim 0.1$, the perturbation to the host particles has not yet become large enough to affect the tracer particles; the tracer diffusion is Gaussian in this regime and the MSDs are linear. As time increases the host matrix becomes more heterogeneous and the tracer MSDs become sublinear, implying transient subdiffusive tracer motion. Figure $3 a$ shows the effect on the MSD of varying the initial host concentration. At volume fractions below $\phi_{1}^{0} \approx 0.55$ the MSDs are approximately linear at all times, similar to observations of anomalous yet Brownian motion [5,6]. At higher host volume fractions above $\phi_{1}^{0}=0.59$, however, the MSDs become increasingly sublinear, similar to experimental observations of tracer subdiffusion near the glass transition $[3,4,8,10]$. 

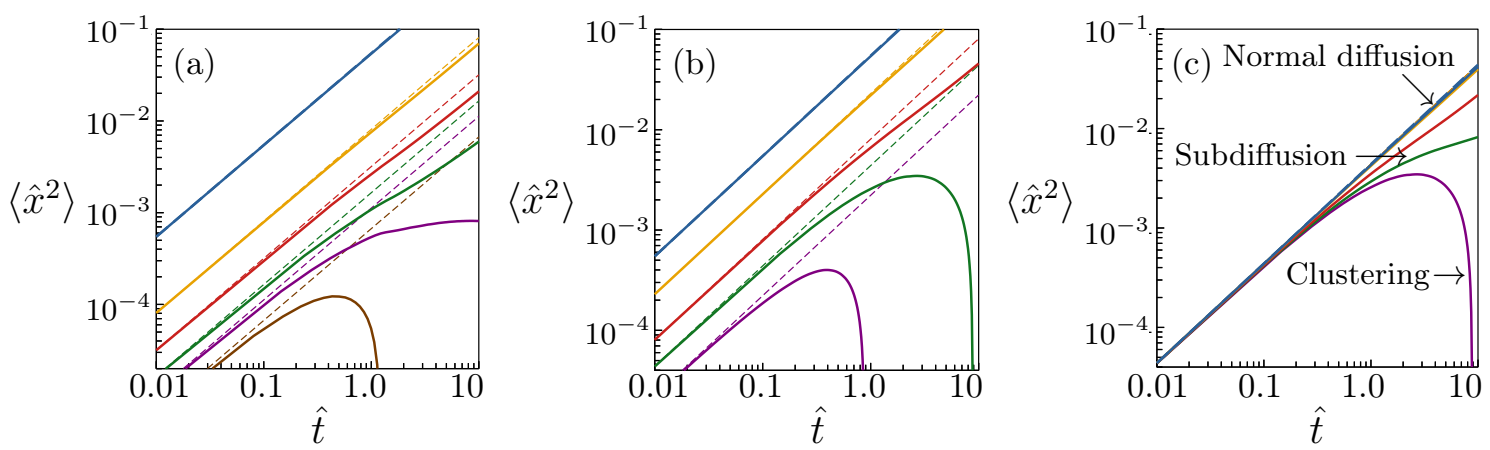

Figure 3: (a) Mean squared displacement $\left\langle\hat{x}^{2}\right\rangle$ computed from equation (16) for the case $\phi_{2}^{0}=0.01$, $\lambda=0.75$ and several initial host matrix volume fractions $\left(\phi_{1}^{0}=0.55\right.$ (blue); 0.59 (orange); 0.60 (red); 0.605 (green); 0.607 (purple); 0.61 (brown)). (b) Mean squared displacements for the case $\phi_{1}^{0}=0.55$ and $\phi_{2}^{0}=0.01$ and several particle size ratios $(\lambda=0.75$ (blue); 0.85 (orange); 0.95 (red); 1.0 (green); 1.05 (purple)). (c) Mean squared displacements for the case $\phi_{1}^{0}=0.55, \lambda=1$ and several tracer concentrations $\left(\phi_{2}^{0}=0.0005\right.$ (blue); 0.001 (orange); 0.005 (red); 0.008 (green); 0.01 (purple)). The dashed lines in (a)-(c) are the Gaussian MSDs $\left\langle\hat{x}^{2}\right\rangle=2 \hat{D}_{22} \hat{t}$ obtained in the absence of cross-diffusion effects.

\subsubsection{Clustering}

Above $\phi_{1}^{0} \approx 0.607$ the tracer particles near the origin exhibit clustering; they move at times $\hat{t}>1$ in a direction opposite to their own concentration gradient (uphill diffusion) and the MSD becomes negative (brown curve in figure 3a). Similarly to cross-diffusion in molecular systems [24], the uphill diffusion is caused by the interplay between the normal diffusion term and the cross-diffusion term on the right-hand-side of the tracer conservation equation $(12 b)$. For relatively low host volume fractions $\phi_{1}$ the Fickian $\hat{D}_{22}$ term dominates and $\partial \phi_{2} / \partial \hat{t}<0$ everywhere in the system. At host concentrations above the glass transition $\phi_{g}=0.58$, however, the tracer diffusivity $\hat{D}_{22}$ approaches zero and the cross-diffusion $\hat{D}_{21}$ term begins to dominate, so that $\partial \phi_{2} / \partial \hat{t}>0$ near the origin. Another way of interpreting the phenomenon is via the colloidal reflection coefficient $\sigma$ given by equation $(6 a)$, which approaches 1 as the host volume fraction increases above $\phi_{g}[18]$. Therefore the host particles have a filtration effect on the tracer particles, pushing them out of the high $\phi_{1}$ regions into the less-concentrated zone at the origin. This effect of the colloidal reflection coefficient on the tracer diffusion provides a potential physical mechanism for the filtration behaviour observed in Brownian dynamics simulations of concentrated hard-sphere suspensions by Ariza and Puertas [25]. Clustering of the tracer particles has also been observed in the molecular dynamics simulations of Làzaro-Làzaro et al. [26], and experimentally by Sentjabrskaja et al. [10] - these works show normal Gaussian tracer diffusion at low host concentrations leading to subdiffusion and finally clustering of the tracer particles at host-matrix concentrations near the glass transition.

\subsubsection{Effect of varying the tracer size and concentration}

Figure $3 b$ illustrates the effect of varying the tracer/host size ratio $\lambda=R_{2} / R_{1}$ in a suspension with $\phi_{1}^{0}=0.55$ and $\phi_{2}^{0}=0.01$. For small tracer particles $(\lambda<0.8)$ the particles can diffuse throughout the pore space; the non-Fickian cross-diffusion term in $(12 b)$ is small relative to the Fickian diffusion term, and the tracer MSDs are linear. As the tracer size increases the diffusion becomes more significantly affected by the host matrix; the non-Fickian term in (12b) begins to control the tracer motion while the Fickian term approaches zero. The tracer MSD becomes increasingly sublinear, leading again to uphill diffusion and clustering at a critical size ratio $\lambda_{c} \approx 1$. A similar transition sequence also occurs by varying the initial tracer concentration $\phi_{2}^{0}$. Figure $3 c$ shows the effect of increasing the tracer concentration in a suspension with $\phi_{1}^{0}=0.55$ and $\lambda=1$, so that the tracer particles are of the same size as the host particles (intra-diffusion). In this case also there is a transition from normal diffusion at sufficiently low tracer concentrations to subdiffusion and finally clustering as the tracer concentration increases above $\phi_{2}^{0}=0.01$. 
Together these results are suggestive of a dynamic phase transition initiated by a subcritical instability of the uniform suspension. That is, in the $\lambda=1$ case the Gaussian tracer pulse plays the role of a small concentration perturbation to the initially uniform host matrix. For sufficiently dilute tracer concentrations or sufficiently low host-matrix concentrations $\phi_{1}^{0} \ll \phi_{g}$ the perturbation decays with time and the suspension is stable. However, at a critical host concentration near to or above the glass transition $\phi_{g}$ the perturbation tends to grow with time. (The total volume fraction $\phi_{T}=\phi_{1}+\phi_{2}$ always decreases with time and is stable.) The possibility that near $\phi_{g}$ the host matrix is unstable to concentration fluctuations is explored further in Section 5.

\subsection{Discussion}

The results in figure 2 depend on the phenomenological expressions for the $D_{i j}$ described in Section 2.1, and in order to have confidence in them it is necessary to test the expressions for the diffusion coefficients at the high concentrations studied. Previously it has been shown that the expression $(2 d)$ for the tracer Fickian diffusivity $D_{22}$ is in good quantitative agreement with experimental data at volume fractions from dilute up to very near the glass transition $\phi_{g}=0.58$ and for several values of the size ratio $\lambda$; also the predicted value of $\phi_{1}$ at which $D_{22} \rightarrow 0$ is in agreement with experiment both for the case of stationary host particles and mobile particles [18]. The equations for the cross-coefficients $D_{12}$ and $D_{21}$ have not yet been tested for hard spheres as cross-diffusion data are not presently available. Measured cross-diffusion coefficients in polymer solutions that interact mainly via excluded-volume effects, however, show a qualitatively similar concentration dependence [27-30]. One significant shortcoming of the expressions for $D_{12}$ and $D_{21}$ is the assumption that the equilibrium partition coefficient $\alpha$ can be written in the form of equation (5a), which is strictly valid only in the dilute limit $\phi_{1} \ll 1[18]$. However, it is shown in the Appendix that in the case $\lambda \leq 1$ the equation gives qualitatively correct results at concentrations up to the close-packed limit.

The anomalous diffusion results in figure 2 are nevertheless a very robust feature of the crossdiffusion model. Qualitatively similar results are obtained, for example, using the more tractable expressions

$$
\begin{gathered}
D_{11}=D_{1}^{0}\left(1-\phi_{1} / \phi_{p}\right)^{-2}, \quad D_{12}=a D_{1}^{0} \phi_{1}, \\
D_{21}=b D_{2}^{0} \phi_{2}, \quad D_{22}=D_{2}^{0}\left(1-\phi_{1} / \phi_{g}\right)^{2} .
\end{gathered}
$$

In these equations $D_{11}$ is in a form used by Davis and Russel [31] while the form of $D_{22}$ was suggested by Rallison [32], and $D_{12}$ and $D_{21}$ with $a=b=3.5$ were obtained by Batchelor [17] in the case $\lambda=1$. These equations satisfy the necessary condition for anomalous diffusion to occur within the model - the tracer Fickian diffusivity $D_{22}$ approaches zero as $\phi_{1} \rightarrow \phi_{g}$ while $D_{11}, D_{12}$ and $D_{21}$ remain finite.

\subsubsection{Dynamic light scattering}

The tracer mean squared displacements in figure 3 are qualitatively similar to those observed in experimental studies and molecular dynamics simulations of anomalous diffusion in hard-sphere suspensions $[3,4,6,8,10,13,26,33]$. The physical situations, however, are similar but not equivalent. Equations (11) simulate the evolution of a macroscopic concentration distribution within the suspension, while particle tracking experiments and light scattering studies typically explore the motion of individual particles or microscopic concentration fluctuations in a macroscopically uniform system $[3,6]$. For normal Gaussian diffusion the MSD from either approach is the same; however, in anomalous systems this is no longer the case [34]. Related to this issue is the different interpretations of gradient diffusion experiments and dynamic light scattering results [35-39].

In dynamic light scattering and particle tracking measurements the particles are observed to undergo Gaussian diffusion at early times, reflecting cage-rattling, and non-Gaussian diffusion at later times, via hopping from cage to cage $[3,32]$. The continuum model developed here does not capture particle-scale effects such as cage rattling, but simulates the evolution of a macroscopic particle concentration distribution. The similarity of the tracer diffusion results to light scattering measurements of the MSD suggests there may nevertheless be an underlying connection between 
cage dynamics and the development of a macroscopic host-matrix perturbation [16]. The effect of concentration perturbations on the stability of a uniform suspension is explored further in the next section.

\section{$5 \quad$ Linear stability analysis}

In linear stability analysis a uniform suspension with initial concentrations $n_{1}^{0}$ and $n_{2}^{0}$ is perturbed by small sinusoidal fluctuations in the form of normal modes, such that

$$
n_{1}=n_{1}^{0}+a_{1} \mathrm{e}^{\omega t} \sin k x \text { and } n_{2}=n_{2}^{0}+a_{2} \mathrm{e}^{\omega t} \sin k x
$$

where $k$ and $\omega$ are the wave number and growth rate of the perturbation and $a_{1}, a_{2}$ are the amplitudes $[35,40,41]$. If the growth rate $\omega$ is less than zero the perturbations decay with time and the suspension is stable, while if $\omega>0$ the perturbations grow and the system is unstable; when $\omega=0$ the suspension is at marginal stability.

For sufficiently small perturbations $\left(a_{1}, a_{2} \ll n_{1}^{0}, n_{2}^{0}\right)$ the diffusion coefficients $D_{i j}$ can be taken as constants $[42,43]$. Inserting (18) into (11) then gives

$$
\omega a_{1}=-k^{2} a_{1} D_{11}-k^{2} a_{2} D_{12} \quad \text { and } \quad \omega a_{2}=-k^{2} a_{1} D_{21}-k^{2} a_{2} D_{22} .
$$

Eliminating the amplitudes $a_{1}$ and $a_{2}$ from (19) and solving the resulting quadratic equation for $\omega$ gives the solutions

$$
\omega_{C}=-k^{2} D_{C}^{L} \quad \text { and } \quad \omega_{S}=-k^{2} D_{S}^{L},
$$

where

$$
D_{C}^{L}=\frac{1}{2}\left[\left(D_{11}+D_{22}\right)+\sqrt{\left(D_{11}+D_{12}\right)^{2}-4\left(D_{11} D_{22}-D_{12} D_{21}\right)}\right],
$$

and

$$
D_{S}^{L}=\frac{1}{2}\left[\left(D_{11}+D_{22}\right)-\sqrt{\left(D_{11}+D_{22}\right)^{2}-4\left(D_{11} D_{22}-D_{12} D_{21}\right)}\right],
$$

are the long-time collective and self diffusion coefficients measured by dynamic light scattering experiments $[35,37,43]$. Setting the growth rate $\omega_{S}$ to zero in equation $(20)$ shows that marginal stability occurs when $D_{S}^{L}=0$. Light scattering experiments showing that $D_{S}^{L} \rightarrow 0$ as the glass transition is approached $[3,44]$ suggest that an initially uniform suspension is therefore only marginally stable to the growth of concentration perturbations.

Requiring $D_{S}^{L} \geq 0$ for stability in $(21 b)$ gives

$$
D_{11} D_{22}-D_{12} D_{21} \geq 0
$$

which is equivalent to the well-known thermodynamic equilibrium stability condition $[35,45,46]$, but here represents a kinetic instability that has been obtained independently of thermodynamic considerations. A kinetic instability could potentially play a role in the formation of the normal modes and long-range concentration fluctuations observed in hard-sphere suspensions near the glass transition $[4,10,26,47,48]$.

\section{Conclusions}

Phenomenological equations describing diffusion of spherical tracer particles in concentrated hardsphere suspensions have been solved numerically. A pulse of tracer particles added to an initially homogeneous suspension perturbs the host matrix via cross diffusion. Depending on the initial host concentration, several distinct transitions of the tracer diffusion occur. At low concentrations, the diffusion is Fickian and Gaussian. At higher concentrations the diffusion becomes nonGaussian, while the tracer mean squared displacement remains approximately linear. Next the mean squared displacement becomes increasingly sublinear, and eventually a kinetic transition occurs in which the tracer particles move in opposition to their own concentration gradient. The origin of the anomalous diffusion is cross-diffusion effects between the host suspension and tracer particles, which become more significant as the tracer Fickian diffusivity decreases near the glass transition. 
At high concentrations the host suspension acts like a dynamic membrane, filtering the tracer particles out of the more crowded regions of the host matrix. Similar transitions also occur by varying the size or concentration of the tracer particles, at a fixed host volume fraction. The theory reproduces several qualitative aspects of anomalous diffusion, including non-Gaussian distribution functions, subdiffusive mean squared displacements and clustering near the glass transition. The perturbations to both the tracer and host particle concentration profiles have been simulated, giving a macroscopic perspective on the physical mechanisms involved. A linear stability analysis suggests that the glass transition is marginally stable to concentration perturbations, yielding a possible mechanism for the onset of normal-mode like dynamic and structural heterogeneity.

\section{Acknowledgements}

This work was made possible by the love and support of Jesus, Lord of heaven and earth and Saviour of all who believe. Believe on the Lord Jesus Christ and thou shalt be saved. Acts 16:31. I would seek unto God, and unto God would I commit my cause: Which doeth great things and unsearchable; marvellous things without number. Job 5:8,9.

\section{A Tracer partition coefficient in concentrated suspensions}

In previous work an expression for the partition coefficient $\alpha$ of tracer particles in a hard-sphere suspension was obtained in the form of equation (5a) [18]. This equation is strictly valid only in the dilute limit, but in practice can be applied at higher concentrations [30,49]. Here, a more general approach is used to obtain an expression for $\alpha$ in concentrated suspensions. In order to introduce necessary concepts a brief review of the previous derivation is first given below.

Figure 4 shows an osmotic equilibrium set-up for a binary hard-sphere suspension. The suspension in compartment A has particle number densities $n_{1}$ and $n_{2}$, and total osmotic pressure $\Pi_{T}$; the suspension is in equilibrium across a semi-permeable partition with a reservoir (compartment B) containing only the fluid solvent and tracer particles at number density $n_{r}$ and osmotic pressure $\pi$. In the McMillan-Mayer theory of suspensions the solvent is assumed incompressible and composed of molecules much smaller than the hard-sphere colloidal particles, so that thermodynamically the system can be treated like a binary hard-sphere gas, with the total osmotic pressure $\Pi_{T}$ of the suspension playing the same role as the total pressure of the gas $[17,50,51]$. In this case the Gibbs-Duhem equation of the mixture can be written as, at constant temperature,

$$
n_{1} \mathrm{~d} \mu_{1}+n_{2} \mathrm{~d} \mu_{2}=\mathrm{d} \Pi_{T}
$$

where $\mu_{i}\left(n_{1}, n_{2}\right)$ is the chemical potential per particle of component $i$. Similarly, the Gibbs-Duhem equation applied to the suspension of tracer particles in compartment B is

$$
n_{r} \mathrm{~d} \mu_{r}=\mathrm{d} \pi,
$$

where $\mu_{r}$ is the chemical potential per particle in the reservoir. At equilibrium the tracer chemical potentials are equal and $\mu_{2}=\mu_{r}$.

\section{A.1 Osmotic pressures}

\section{A.1.1 Host particles}

The host-particle osmotic pressure in $\mathrm{A}$ is defined as the difference between the pressures of compartments $\mathrm{A}$ and $\mathrm{B}$, so that $\Pi=\Pi_{T}-\pi[18,50]$. In the tracer limit $\phi_{2} \rightarrow 0$, $\Pi$ depends only on $n_{1}$ and can be written as

$$
\Pi=n_{1} k_{B} T Z,
$$

where $Z\left(\phi_{1}\right)$ is the hard-sphere compressibility factor given approximately by $(3 b)$. While $(3 b)$ suffices for the qualitative results obtained in Section 4 , here a more accurate equation of state for 


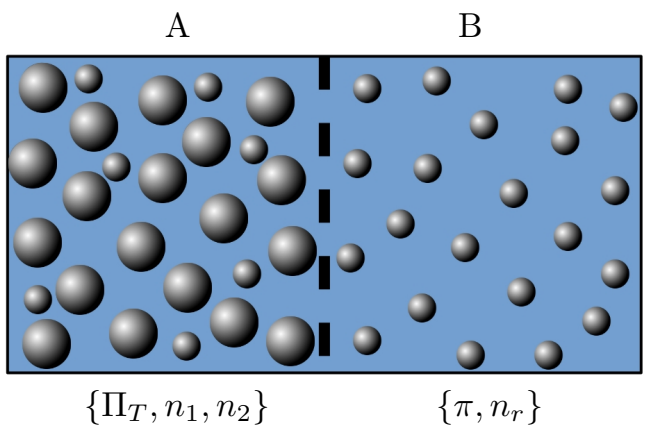

Figure 4: Illustration of osmotic equilibrium in a bidisperse hard-sphere suspension. The membrane separating compartments A and B is permeable to the fluid (blue) and smaller tracer particles but impermeable to the larger host particles. Because of excluded volume effects, the equilibrium tracer concentration $n_{2}$ in compartment $\mathrm{A}$ is less than the concentration $n_{r}$ in the reservoir compartment $\mathrm{B} ; \alpha=n_{2} / n_{r}$ is the equilibrium partition coefficient. $\Pi_{T}$ is the total osmotic pressure of the mixture in A, while $\pi$ is the osmotic pressure of the tracer suspension in B.

$Z$ is used in order to obtain an accurate equation for $\alpha$. At host volume fractions below $\phi_{1} \approx 0.55$, the Carnahan-Starling equation

$$
Z=\frac{1+\phi_{1}+\phi_{1}^{2}-\phi_{1}^{3}}{\left(1-\phi_{1}\right)^{3}}
$$

agrees very closely (to within $1 \%$ ) with the results of molecular dynamics simulations $[52,53]$. However, equation (26) becomes inaccurate as $\phi_{1}$ increases above the glass transition $\phi_{g}=0.58$. At volume fractions above 0.55 the compressibility factor is more accurately given by the Speedy equation of state $Z=2.67 /\left(1-\phi / \phi_{p}\right)$ [54]. The following empirical equation

$$
Z=\frac{1+a \phi_{1}+32 \phi_{1}^{3}-55 \phi_{1}^{4}}{\left(1-\phi_{1} / \phi_{p}\right)},
$$

where $a=4-1 / \phi_{p}$, agrees to within $5 \%$ with the results of molecular dynamics simulation data at volume fractions up to 0.55 , and at higher volume fractions is of comparable accuracy to the Speedy equation [18]. More accurate expressions for $Z$ developed by LeFevre [55], Liu [56] or Paricaud [57] for volume fractions above 0.55 could also be used, but equation (27) has a relatively simple form and is of sufficient accuracy for the present purposes.

\section{A.1.2 Tracer particles}

The osmotic pressure $\pi$ of the tracer particles in the reservoir compartment B can be written as

$$
\pi=n_{r} k_{B} T Z\left(\phi_{r}\right)
$$

where $Z\left(\phi_{r}\right)$ is the hard-sphere compressibility factor evaluated at $\phi_{r}=v_{2} n_{r}$. Equation (28) generalizes the corresponding equation for $\pi$ used previously [18] by allowing the tracer concentration in the reservoir to be non-dilute. At high host concentrations $\phi_{1} \rightarrow \phi_{p}$, excluded volume effects cause the tracer concentration $\phi_{r}$ in the reservoir to be non-dilute, even though $\phi_{2}$ in compartment $\mathrm{A}$ is dilute. For the case $\lambda=1$ in the close-packed limit $\phi_{1} \rightarrow \phi_{p}$, the reservoir concentration must also approach close packing $\phi_{r} \rightarrow \phi_{p}$ in order to maintain equilibrium. This phenomenon is discussed in more detail below.

\section{A.2 Equilibrium partition coefficient}

\section{A.2.1 Dilute limit}

In the dilute limit $\phi_{2} \ll \phi_{1} \ll 1$ the chemical potential of the tracer particles in compartment A of figure 4 can be written as

$$
\mu_{2}=\mu_{2}^{0}+k_{B} T \ln \phi_{2}+\mu_{2}^{e x}
$$


where $\mu_{2}^{0}$ is a constant and

$$
\mu_{2}^{e x}=k_{B} T b_{12} \phi_{1}
$$

is the excess chemical potential caused by interparticle interactions [58]. In the limit $\phi_{r} \ll 1$ the tracer chemical potential in compartment B is (for an incompressible fluid)

$$
\mu_{r}=\mu_{2}^{0}+k_{B} T \ln \phi_{r} .
$$

Equating the chemical potentials at local equilibrium, $\mu_{2}=\mu_{r}$, gives the partition coefficient as

$$
\alpha=\frac{\phi_{2}}{\phi_{r}}=\mathrm{e}^{-b_{12} \phi_{1}},
$$

as obtained previously [18]. Using Widom's insertion method Lekkerkerker [50] derived a more general expression for the case $\lambda \ll 1$ valid at semi-dilute concentrations in the form

$$
\alpha=\left(1-\phi_{1}\right) \mathrm{e}^{-\left(A y+B y^{2}+C y^{3}\right)},
$$

where $y=\phi_{1} /\left(1-\phi_{1}\right), A=3 \lambda+3 \lambda^{2}+\lambda^{3}, B=(9 / 2) \lambda^{2}+3 \lambda^{3}$ and $C=3 \lambda^{3}$.

\section{A.2.2 Concentrated suspensions}

In concentrated suspensions the tracer chemical potential in compartment A is

$$
\mu_{2}=\mu_{2}^{0}+k_{B} T \ln \phi_{2}+\mu_{2}^{e x}
$$

where the excess tracer chemical potential is a more complicated function than equation (30). By considering the work required to insert a tracer particle of radius $R_{2}$ into a uniform sea of host particles of radius $R_{1}$ and volume fraction $\phi_{1}$, Heyes and Santos [59] derived an expression for $\mu_{2}^{e x}$, valid at arbitrary host-particle concentrations $\phi_{1}$, in the form

$$
\mu_{2}^{e x}=k_{B} T\left(c_{0}+c_{1} \lambda+c_{2} \lambda^{2}+c_{3} \lambda^{3}\right) .
$$

The coefficients in (34) are functions of $\phi_{1}$ such that

$$
c_{0}=-\ln \left(1-\phi_{1}\right), \quad c_{1}=\frac{3 \phi_{1}}{1-\phi_{1}}, \quad c_{2}=c_{1}+3 a_{2}, \quad c_{3}=Z-1-\frac{1}{3} c_{1}-\frac{2}{3} c_{2},
$$

where $Z$ is the compressibility factor,

$$
a_{2}=\int_{0}^{\phi_{1}}\left(\frac{Z^{*}}{\phi_{1}}\right) \mathrm{d} \phi_{1}
$$

and $Z^{*}=Z-\left(1+2 \phi_{1}\right) /\left(1-\phi_{1}\right)^{2}$. In the dilute limit $\phi_{1} \ll 1$, equation (34) gives $\mu_{2}^{e x}=$ $k_{B} T(1+\lambda)^{3} \phi_{1}$, and agrees with (30). Integrating (36) with equation (27) for $Z$ gives $^{1}$

$$
a_{2}=1.311 \phi_{1}+1.024 \phi_{1}^{2}+11.733 \phi_{1}^{3}-1.721 \ln \left(1-\phi_{1} / \phi_{p}\right)-\left(c_{0}+c_{1}\right) .
$$

The chemical potential of the particles in compartment B can be obtained by integrating equation (24), using (28) for $\pi$, giving

$$
\mu_{r}=\mu_{2}^{0}+k_{B} T \int_{0}^{\phi_{r}} \frac{1}{\phi_{r}} \frac{\mathrm{d}\left(\phi_{r} Z\right)}{\mathrm{d} \phi_{r}} \mathrm{~d} \phi_{r} .
$$

Inserting $Z\left(\phi_{r}\right)=\left(1+a \phi_{r}+32 \phi_{r}^{3}-55 \phi_{r}^{4}\right) /\left(1-\phi_{r} / \phi_{p}\right)$ into (38) and integrating gives ${ }^{2}$

$$
\mu_{r}=\mu_{2}^{0}+k_{B} T \ln \phi_{r}+\mu_{r}^{e x},
$$

\footnotetext{
${ }^{0}$ To first order in $\phi_{2}, \mu_{2}^{e x} \approx 8 k_{B} T \phi_{2}+\mu_{2}^{i n}\left(T, \phi_{1}\right)$.

${ }^{1}$ Alternatively, using the Carnahan-Starling equation (26) gives $a_{2}=-c_{0}-\phi_{1} /\left(1-\phi_{1}\right)^{2}$.

${ }^{2}$ Using the Carnahan-Starling equation for $Z$ in (38) gives $\mu_{r}^{e x}=k_{B} T \phi_{r}\left(8-9 \phi_{r}+3 \phi_{r}^{2}\right) /\left(1-\phi_{r}\right)^{3}$; to first order in $\phi_{r}$ this becomes $\mu_{r}^{e x}=8 k_{B} T \phi_{r}$.
} 
where

$$
\frac{\mu_{r}^{e x}}{k_{B} T}=2.621 \phi_{r}+3.072 \phi_{r}^{2}+46.933 \phi_{r}^{3}-1.721 \ln \left(1-\phi_{r} / \phi_{p}\right)+\frac{2.689 \phi_{r}}{\left(1-\phi_{r} / \phi_{p}\right)} .
$$

To first order in $\phi_{r}$ equation (40) gives $\mu_{r}^{e x}=8 k_{B} T \phi_{r}$.

Equating the chemical potentials in (33) and (39) gives the partition coefficient as

$$
\alpha \equiv \frac{\phi_{2}}{\phi_{r}}=\mathrm{e}^{-\left(\mu_{2}^{e x}-\mu_{r}^{e x}\right) / k_{B} T}
$$

Equation (41) is a nonlinear equation for $\alpha$ that can be solved numerically using Matlab's fzero function (Matlab files included as Supplementary Material). Figure 5 shows equations (41) (solid blue curves), (32) (dashed red curves) and (31) (dash-dotted green curves) plotted versus $\phi_{1}$ for the case $\phi_{2}=0.001$ and several values of $\lambda$. When $\lambda=0.03$, Lekkerkerker's [50] expression (32) is in good quantitative agreement with the full model at all concentrations up to the close-packed limit $\phi_{1}=\phi_{p}$. Equation (31) gives qualitatively correct results for $\alpha$, though somewhat overestimates the actual value.

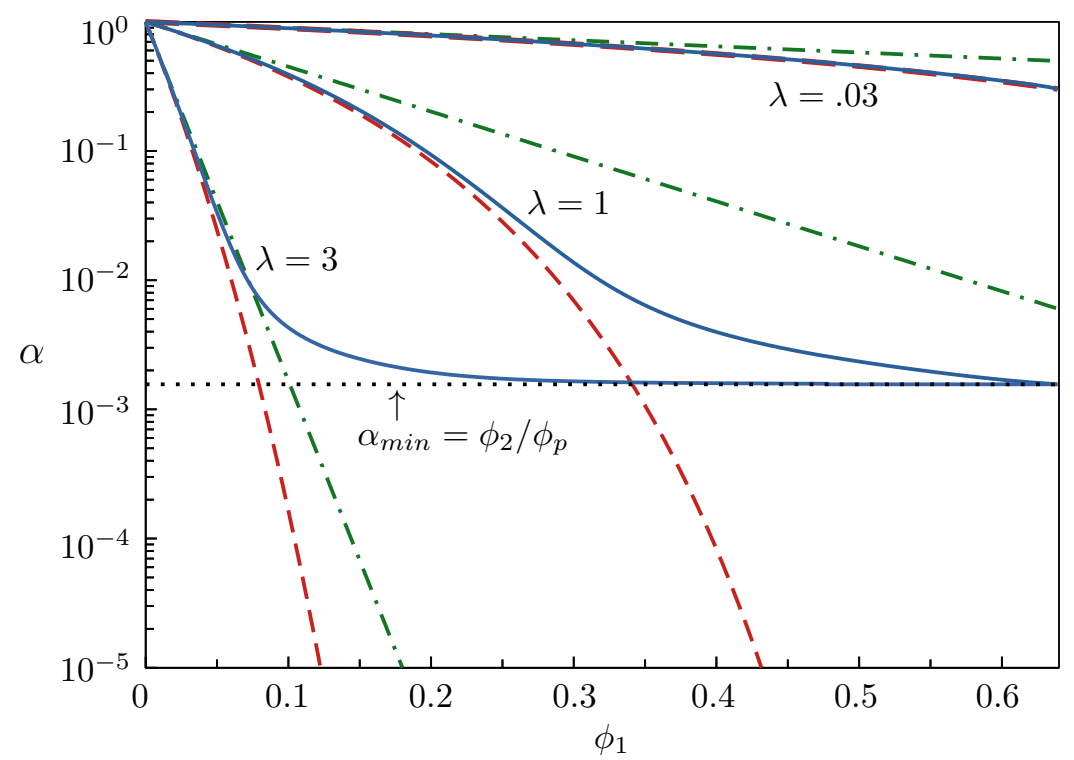

Figure 5: The equilibrium partition coefficient $\alpha$ plotted verses $\phi_{1}$ for the case $\phi_{2}=.001$ and several values of $\lambda$. The blue solid curves are from equation (41); the red dashed curves are from Lekkerkerker's expression (32); the green dot-dashed curves are from (31).

For $\lambda=1$, equation (31) gives qualitatively correct results but again overestimates the actual value of $\alpha$. Lekkerkerker's expression (32) is in good agreement with (41) at concentrations up to $\phi_{1} \approx 0.3$; at higher concentrations $\phi_{1}>0.4$ it gives a value for $\alpha$ that is several orders of magnitude too small as $\phi_{1} \rightarrow \phi_{p}$. As $\phi_{1}$ increases in the $\lambda=1$ case the partition coefficient $\alpha$ approaches the minimum possible value for hard spheres, $\alpha_{\min }=\phi_{2} / \phi_{p}$, shown as the dotted black line in figure 5 . In the close-packed limit $\phi_{1} \rightarrow \phi_{p}$ the tracer particles in compartment A are jammed in between the host particles, and in order to maintain equilibrium the tracer particles in compartment B must also approach the close-packing concentration $\phi_{r} \rightarrow \phi_{p}$. For hard spheres the maximum possible concentration in the reservoir is $\phi_{p}$, and the minimum possible value of the partition coefficient is therefore $\alpha_{\text {min }}=\phi_{2} / \phi_{p}$. For $\lambda \geq 1$ the full solution (41) approaches this minimum as $\phi_{1}$ increases. An approximate explicit expression for $\alpha$ that has the correct limits is

$$
\alpha=\left(1-\phi_{2} / \phi_{p}\right) \mathrm{e}^{-b_{12} \phi_{1}}+\phi_{2} / \phi_{p}
$$

For the case $\lambda=3$ both equations (31) and (32) break down beyond the dilute limit, and either (41) or (42) should be used in concentrated suspensions when $\lambda>1$. 


\section{References}

[1] Ralf Metzler. Brownian motion and beyond: first-passage, power spectrum, non-Gaussianity, and anomalous diffusion. Journal of Statistical Mechanics: Theory and Experiment, 2019, 114003, 2019.

[2] Fernando A. Oliveira, Rogelma M. S. Ferreira, Luciano C. Lapas, and Mendeli H. Vainstein. Anomalous diffusion: A basic mechanism for the evolution of inhomogeneous systems. Frontiers in Physics, 7, 00018, 2019.

[3] W. van Megen, T. C. Mortensen, S. R. Williams, and J. Müller. Measurement of the selfintermediate scattering function of suspensions of hard spherical particles near the glass transition. Phys. Rev. E, 58, 6073-6085, 1998.

[4] W. K. Kegel and A. van Blaaderen. Direct observation of dynamical heterogeneities in colloidal hard-sphere suspensions. Science, 287, 290-293, 2000.

[5] B. Wang, J. Kuo, S. C. Bae, and S. Granick. When Brownian diffusion is not Gaussian. Nature Materials, 11, 481-485, 2012.

[6] J. Guan, B. Wang, and S. Granick. Even hard-sphere colloidal suspensions display Fickian yet non-Gaussian diffusion. ACS Nano, 8, 3331-3336, 2014.

[7] George David Joseph Phillies. In complex fluids the Gaussian diffusion approximation is generally invalid. Soft Matter, 11, 580-586, 2015.

[8] Alice L. Thorneywork, Dirk G. A. L. Aarts, Jürgen Horbach, and Roel P. A. Dullens. On the Gaussian approximation in colloidal hard sphere fluids. Soft Matter, 12, 4129-4134, 2016.

[9] W. He, H. Song, Y. Su, L. Geng, B. J. Ackerson, H. B. Peng, and P. Tong. Dynamic heterogeneity and non-Gaussian statistics for acetylcholine receptors on live cell membrane. Nature Communications, 7, 11701, 2016.

[10] T. Sentjabrskaja, E. Zaccarelli, C. De Michele, F. Sciortino, P. Tartaglia, T. Voigtmann, S. U. Egelhaaf, and M. Laurati. Anomalous dynamics of intruders in a crowded environment of mobile obstacles. Nature Communications, 7, 11133, 2016.

[11] Thomas J. Lampo, Stella Stylianidou, Mikael P. Backlund, Paul A. Wiggins, and Andrew J. Spakowitz. Cytoplasmic RNA-protein particles exhibit non-Gaussian subdiffusive behavior. Biophysical Journal, 112, 532 - 542, 2017.

[12] M. Wolfson, C. Liepold, B. Lin, and S. A. Rice. A comment on the position dependent diffusion coefficient representation of structural heterogeneity. The Journal of Chemical Physics, 148, 194901, 2018.

[13] Tatjana Sentjabrskaja, Alan R. Jacob, Stefan U. Egelhaaf, George Petekidis, Thomas Voigtmann, and Marco Laurati. Binary colloidal glasses: linear viscoelasticity and its link to the microscopic structure and dynamics. Soft Matter, 15, 2232-2244, 2019.

[14] Ryan C. Roberts, Ryan Poling-Skutvik, Jeremy C. Palmer, and Jacinta C. Conrad. Tracer transport probes relaxation and structure of attractive and repulsive glassy liquids. The Journal of Physical Chemistry Letters, 9, 3008-3013, 2018.

[15] C Patrick Royall, Francesco Turci, Soichi Tatsumi, John Russo, and Joshua Robinson. The race to the bottom: approaching the ideal glass? Journal of Physics: Condensed Matter, 30, $363001,2018$.

[16] W. van Megen and H. J. Schöpe. The cage effect in systems of hard spheres. The Journal of Chemical Physics, 146, 104503, 2017.

[17] G. K. Batchelor. Diffusion in a dilute polydisperse system of interacting spheres. Journal of Fluid Mechanics, 131, 155-175; and Corrigendum 137, 1983, 467-469, 1983. 
[18] S. S. L. Peppin. Theory of tracer diffusion in concentrated hard-sphere suspensions. J. Fluid Mech., 870, 1105-1126, 2019.

[19] M. G. Worster, S. S. L. Peppin, and J. S. Wettlaufer. Colloidal mushy layers. Journal of Fluid Mechanics, 914, A28, 2021.

[20] G. K. Batchelor. Sedimentation in a dilute dispersion of spheres. Journal of Fluid Mechanics, 52, 245-268, 1972.

[21] W. B. Russel, D. A. Seville, and W. R. Schowalter. Colloidal Dispersions. Cambridge University Press, U.K., 1989.

[22] R. B. Bird, W. E. Stuart, and E. N. Lightfoot. Transport Phenomena Second Edition. Wiley, N.Y., 2002.

[23] W. E. Scheisser and G. W. Griffiths. A Compendium of Partial Differential Equation Models: Method of Lines Analysis with Matlab. Cambridge University Press, U.K., 2009. https://www.lehigh.edu/ wes1/.

[24] Rajamani Krishna. Uphill diffusion in multicomponent mixtures. Chem. Soc. Rev., 44, 2812-2836, 2015.

[25] M. J. Ariza and A. M. Puertas. Colloidal permeability of liquid membranes consisting of hard particles by nonequilibrium simulations. The Journal of Chemical Physics, 131, 164903, 2009.

[26] E. Làzaro-Làzaro, J. A. Moreno-Razo, and M. Medina-Noyola. Anomalous dynamic arrest of non-interacting spheres ("polymer") diluted in a hard-sphere ("colloid") liquid. The Journal of Chemical Physics, 148, 104505, 2018.

[27] P. Daivis, I. Snook, W. Van Megen, B. N. Preston, and W. D. Comper. Dynamic light scattering measurements of diffusion in polymer-polymer-solvent systems. Macromolecules, 17, 2376-2380, 1984.

[28] A. Vergara, O. Annunziata, L. Paduano, D. G. Miller, J. G. Albright, and R. Sartorio. Multicomponent diffusion in crowded solutions. 2. Mutual diffusion in the ternary system tetra(ethylene glycol)-NaCl-water. The Journal of Physical Chemistry B, 108, 2764-2772, 2004.

[29] O. Annunziata, J. A. Rard, J. G. Albright, L. Paduano, and D. G. Miller. Mutual diffusion coefficients and densities at $298.15 \mathrm{~K}$ of aqueous mixtures of $\mathrm{NaCl}$ and $\mathrm{Na} 2 \mathrm{So} 4$ for six different solute fractions at a total molarity of $1.500 \mathrm{~mol} \cdot \mathrm{dm}^{-3}$ and of aqueous Na2SO4. Journal of Chemical \& Engineering Data, 45, 936-945, 2000.

[30] S. S. L. Peppin. Effective hard-sphere model of diffusion in aqueous polymer solutions. Open Science Foundation Preprints, https://osf.io/9yuje, 2021.

[31] K. E. Davis and W. B. Russel. An asymptotic description of transient settling and ultrafiltration of colloidal dispersions. Physics of Fluids A: Fluid Dynamics, 1, 82-100, 1989.

[32] J. M. Rallison. Brownian diffusion in concentrated suspensions of interacting particles. Journal of Fluid Mechanics, 186, 471-500, 1988.

[33] S. D. W. Hannam, P. J. Daivis, and G. Bryant. Dramatic slowing of compositional relaxations in the approach to the glass transition for a bimodal colloidal suspension. Phys. Rev. E, 96, 022609, 2017.

[34] R. Metzler, J.-H. Jeon, A. G. Cherstvy, and E. Barkai. Anomalous diffusion models and their properties: Non-stationarity, non-ergodicity, and ageing at the centenary of single particle tracking. Phys. Chem. Chem. Phys., 16, 24128-24164, 2014. 
[35] George D. J. Phillies. Effects of intermacromolecular interactions on diffusion. II. Threecomponent solutions. The Journal of Chemical Physics, 60, 983-989, 1974.

[36] Derek G. Leaist and Ling Hao. Comparison of diffusion coefficients of multicomponent solutions from light scattering and macroscopic gradient techniques: sodium dodecyl sulfate micelles in aqueous salt solutions. The Journal of Physical Chemistry, 97, 7763-7768, 1993.

[37] Onofrio Annunziata, Daniela Buzatu, and John G. Albright. Protein diffusion coefficients determined by macroscopic-gradient Rayleigh interferometry and dynamic light scattering. Langmuir, 21, 12085-12089, 2005.

[38] Bijan Das, Basanti Maitra, Sean M. Mercer, Michelle Everist, and Derek G. Leaist. A comparison of diffusion coefficients for ternary mixed micelle solutions measured by macroscopic gradient and dynamic light scattering techniques. Phys. Chem. Chem. Phys., 10, 3083-3092, 2008.

[39] George D. J. Phillies. Interpretation of quasielastic scattering spectra of probe species in complex fluids. The Journal of Chemical Physics, 139, 034902, 2013.

[40] S. Chandrasekhar. Hydrodynamic and Hydromagnetic Stability. Clarendon, Oxford, 1961.

[41] G. K. Batchelor and J. W. Janse van Rensburg. Structure formation in bidisperse sedimentation. Journal of Fluid Mechanics, 166, 379-407, 1986.

[42] H. L. Toor. Solution of the linearized equations of multicomponent mass transfer: I. AIChE Journal, 10, 448-455, 1964.

[43] P. N. Pusey, H. M. Fijnaut, and A. Vrij. Mode amplitudes in dynamic light scattering by concentrated liquid suspensions of polydisperse hard spheres. The Journal of Chemical Physics, 77, 4270-4281, 1982.

[44] G. L. Hunter and E. R. Weeks. The physics of the colloidal glass transition. Rev. Mod. Phys., 75, 066501, 2012.

[45] J. S. Kirkaldy. Diffusion in multicomponent metallic systems: I. Phenomenological theory for substitutional solid solution alloys. Canadian Journal of Physics, 36, 899-906, 1958.

[46] V. Vitagliano, R. Sartorio, S. Scala, and D. Spaduzzi. Diffusion in a ternary system and the critical mixing point. Journal of Solution Chemistry, 7, 605-622, 1978.

[47] Jennifer M. Lynch, Gianguido C. Cianci, and Eric R. Weeks. Dynamics and structure of an aging binary colloidal glass. Phys. Rev. E, 78, 031410, 2008.

[48] Antina Ghosh, Vijayakumar Chikkadi, Peter Schall, and Daniel Bonn. Connecting structural relaxation with the low frequency modes in a hard-sphere colloidal glass. Phys. Rev. Lett., 107, 188303, 2011.

[49] M. S. McAfee and O. Annunziata. Effect of particle size on salt-induced diffusiophoresis compared to Brownian mobility. Langmuir, 30, 4916-4923, 2014.

[50] H. N. W. Lekkerkerker and A. Stroobants. On the spinodal instability of highly asymmetric hard sphere suspensions. Physica A, 195, 387-397, 1993.

[51] O. Annunziata, A. Vergara, L. Paduano, R. Sartorio, D. G. Miller, and J. G. Albright. Quaternary diffusion coefficients in a protein-polymer-salt-water system determined by Rayleigh interferometry. The Journal of Physical Chemistry B, 113, 13446-13453, 2009.

[52] N. F. Carnahan and K. E. Starling. Equation of state for nonattracting rigid spheres. The Journal of Chemical Physics, 51, 635-636, 1969.

[53] G.-W. Wu and R. J. Sadus. Hard sphere compressibility factors for equation of state development. AIChE Journal, 51, 309-313, 2005. 
[54] Robin J. Speedy. On the reproducibility of glasses. The Journal of Chemical Physics, 100, 6684-6691, 1994.

[55] E. LeFevre. Equation of state for hard-sphere fluid. Nature, 235, 20, 1972.

[56] H. Liu. A very accurate hard sphere equation of state over the entire stable and metstable region. arXiv, cond-mat/0605392, 2006.

[57] P. Paricaud. Extension of the BMCSL equation of state for hard spheres to the metastable disordered region: Application to the SAFT approach. The Journal of Chemical Physics, 143, 044507, 2015.

[58] A. Santos and R. D. Rorhmann. Chemical-potential route for multicomponent fluids. Phys. Rev. E, 87, 052138, 2013.

[59] D. M. Heyes and A. Santos. Chemical potential of a test hard sphere of variable size in a hard-sphere fluid. J. Chem. Phys., 145, 214504, 2016. 